\title{
A review of chemistry and biological activities of the Indonesian Octocorallia
}

\author{
Masteria Yunovilsa Putra, Joko Tri Wibowo, Tutik Murniasih \\ Research Center for Oceanography, Indonesian Institute of Sciences, Jl. Pasir Putih I, Ancol Timur, Jakarta 14430, Indonesia.
}

\section{ARTICLE INFO \\ Article history: \\ Received on: 28/06/2016 \\ Accepted on: 23/08/2016 \\ Available online: $30 / 05 / 2017$}

\section{Key words:}

marine invertebrates, octocorals, secondary metabolites, biological activities.

\begin{abstract}
Marine invertebrates are known to produce secondary metabolites that may have potential as new drug candidates. Numerous chemical studies have shown that sponges are a rich source of marine bioactive compounds. However, recent studies demonstrate that octocorals also produce secondary metabolites with promising pharmacological activities. In this paper, we report on the chemical and biological activities of the Indonesian octocorals, particularly soft corals, gorgonians and sea pens. Octocorals from the Ind onesian coasts have been demonstrated to contain a wide variety of compounds including steroids, sesquiterpenes and diterpenes. Cembranoid diterpenes have been largely founds in gorgonian and soft corals and they are believed to function as chemical defenses. Anti-inflammatory, antimicrobial, cytotoxic to antitumor have been disclosed for members of the cembranoid class.
\end{abstract}

\section{INTRODUCTION}

Marine organisms are an important source of new bioactive molecules; thus the scientific community worldwide is focusing its efforts on the isolation and characterization of biologically active natural products (Almeida et al., 2014). Since the early days of marine natural products research in the 1960s, sponges have yielded the largest number of new metabolites reported per year compared to any other plant or animal phylum known from the marine environment (Edaba and Proksch, 2012). However, with the development of new methods in analytical technology, spectroscopy, and high-throughput screening, the bioprospecting potential of cnidarians as they have also produced secondary metabolites with promising pharmacological activities (Molinski et al., 2009). A total of 3244 marine natural products was described from this phylum alone between 1990 and 2011, which shows the importance of cnidarians for marine natural product research (Rocha et al., 2015).

\footnotetext{
* Corresponding Author
}

Email:mast001@lipi.go.id
The phylum Cnidarian is a large, diverse and ecologically important group of marine invertebrates that are divided into five classes: Anthozoa, Hydrozoa, Cubozoa, Straurozoa and Chyphozoa and it contains over 11000 species, 7500 of them belonging to the class Anthozoa (Rocha et al., 2011; Daly et al., 2007). From the 3244 new compounds yielded by marine Cnidarians since 1990, 99\% were discovered within Anthozoa. The remaining $1 \%$ is associated with species from Hydrozoa (Rocha et al., 2015). Anthozoa is divided into the subclasses Hexacorallia and Octocorallia, each one further separated into multiple orders. Octocorals comprise soft corals, sea pens and gorgonians, with eight tentacles and eight internal mesenteries that exhibit less variation in polyp morphology than hexacorals (Almeida et al., 2014; Rocha et al., 2011; McFadden et al., 2010). Octocorallia comprise approximately 3200 species of soft corals (Alcyonacea) found in all marine environments and 94\% of new compounds from cnidarians were discovered from soft corals or Alcyonacea (Rocha et al., 2015). With all of these compounds, soft corals, or alcyonacea, proved to be the most promising source of compounds for pharmaceutical use.Terpenoid chemistry predominates across the Octocorallia class. 
Sesquiterpenes and diterpenes are the most common terpenes isolated from Octocorallia. Numerous ecological studies have shown that to survive in the complicated marine environment, most octocorals produce diterpenes compounds, and these compounds are generally believed to function as chemical defenses. Several bioactivities including anti-inflammatory (Putra et al., 2012; Kapojos et al., 2010), antimicrobial (Wang et al., 2009), cytotoxic (Rodríguezet al., 1998; Fu et al., 1999), antitumor (Chen et al., 2011) have been isolated from members of the terpenoid class of Octocorallia. Since 1997-2014, more than 22 publications have reported on the bioactive compounds from Indonesian octocorallia such as sea pens, soft corals and gorgonians. Soft corals are the dominant reef-dwelling octocorals in the Indo-Pacific regions including Indonesia. Almost 99\% of new marine natural products from Indonesian octcorals were collected from soft corals. In this review we report the marine compounds isolated from octocorals from Indonesia, specifically focusing on their structures and biological activities.

\section{Soft corals}

Soft corals are rich sources of secondary metabolites, particularly diterpenoids, sesquiterpenoid and steroids. The bioactive compounds in eight species of Indonesian soft corals such as Sinularia sp, Lobophytum sp, Cladiella sp, Xenia sp, Sarcophyton sp, Nephthea sp and Minabea sp, have been reported. Handayani and colleagues isolated two new oxygenated sesquiterpenes, hydroxycolorenone (1) and methoxycolorenone(2), and the known sesquiterpene, (+)-cyclocolorenone (3) from
Nephthea chabrolii collected by snorkelling off the shores of Sinyary Island of West Sumatera (Handayani et al., 1997). Compound 1 exhibited insecticidal activity towards neonate larvae of the polyphagous pest insect Spodoptera littoralis, with an $\mathrm{EC}_{50}$ of $8.8 \mathrm{ppm}$ and $\mathrm{LC}_{50}$ of $453 \mathrm{ppm}$, when incorporated in an artificial diet and offered to larvae in a chronic feeding bioassay.

Januar and colleagues also isolated a new 3, 4-epoxynephthenol acetate (4) along with five known compounds: decaryiol (5), 15-hydroxy-cembrenene (6),2-hydroxy-nephthenol (7), nephthenol (8) and arachidonic acid (9) from two specimens of the soft coral Nephthea sp., collected from the Seribu Islands (Januar et al., 2010). Compounds 4 -9 were screened for their whole cell anticancer activity against three human tumor cell lines; SF-268 (CNS), MCF-7 (breast), H460 (lung). Unfortunately, compounds 4 - 9 demonstrated weak (GI50> $100 \mu \mathrm{M})$ nonselective activity towards the three cell lines. Morris et al. described a new -epoxy-9,11-secogorgostan-9-one $\alpha, 6 \alpha, 11$ Trihydroxy-5 $\beta, 7 \beta 3(\mathbf{1 0})$, with a gorgosterol side chain and an unusual oxygenation pattern on the $\mathrm{A}$ and $\mathrm{B}$ rings from Lobophytum sp., collected at Mayu Island, Molucca Sea (Morris et al., 1998). Fattorusso et al. identified a new member of the family of zoanthamine-type alkaloids named lobozoanthamine (11) and three novel cembrane diterpenoids, decaryiols B-D (12-14), along with three known cembranoids named 2-hydroxy-nephthenol (7), epoxycembrane (15), decaryiol (16) from the Indonesian soft coral Lobopphytum sp., collected along the island of Siladen, in the Bunaken Marine Park near Manado (North Sulawesi, Indonesia) (Fattorusso et al., 2009).

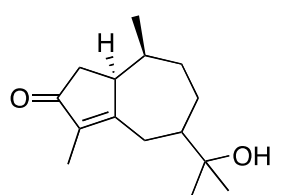

Hydroxycolorenone (1)

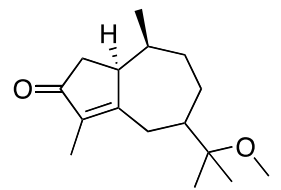

Methoxycolorenone (2)

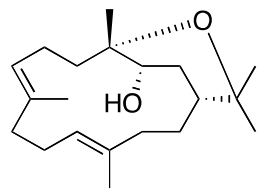

Decaryiol (5)

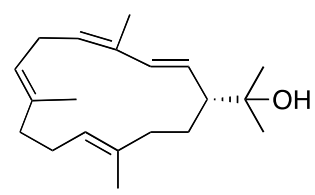

15-Hydroxy-cembrenene (6)

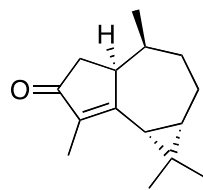

(+)-Cyclocolorenone (3)

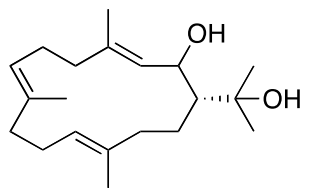

2-Hydroxy-nephthenol (7)

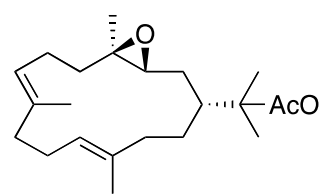

3,4-Epoxy-nephthenol acetate (4)<smiles>CCCCC/C=C/C/C=C/C/C=C/C/C=C/CCCC(=O)O</smiles>

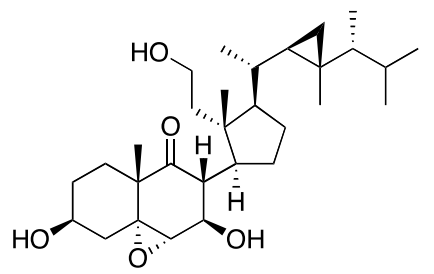

3b,7b,11-Trihydroxy-5a,6a-epoxy-9,11-secogorgostan-9-one (10)

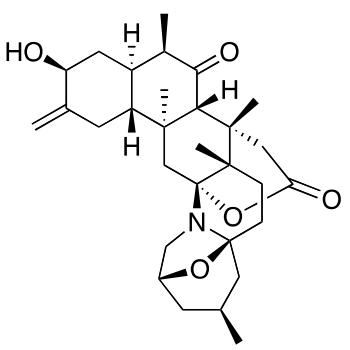

Lobozoanthamine (11)

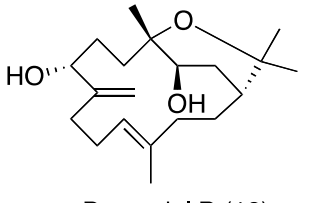

Decaryiol B (12)

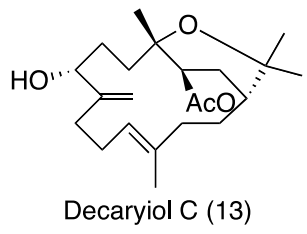




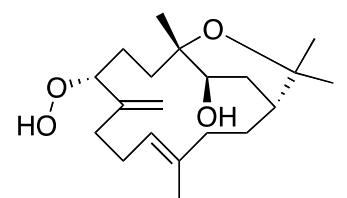

Decaryiol D (14)

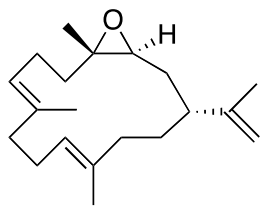

Epoxycembrane (15)

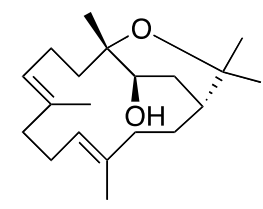

Decaryiol (16)

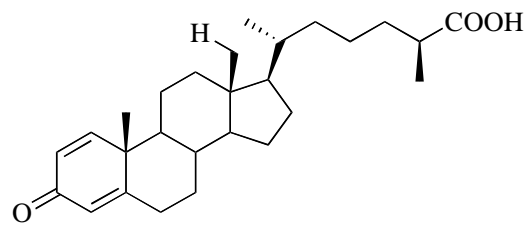

(25S)-3-oxocholesta-1,4-dien-26-oic acid (17)

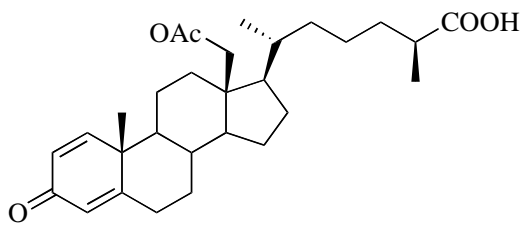

(25S)-18-Acetoxy-3-oxocholesta-1,4-dien-26-oic acid (18)

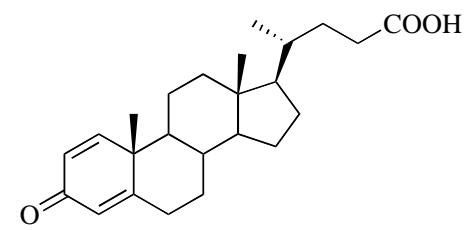

3-Oxochol-1,4-dien-24-oic acid (19)

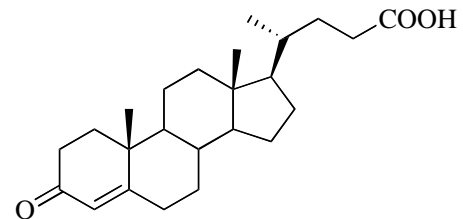

3-Oxochol-4-en-24-oic acid (20)

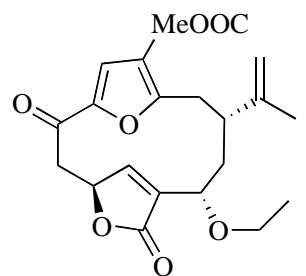

Sarcofuranocembrenolide A (21)

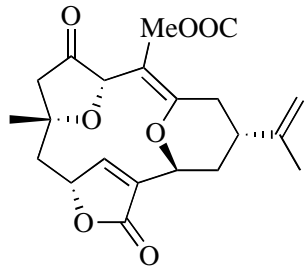

(24)

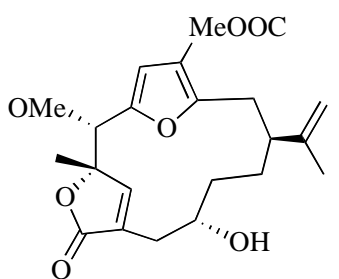

Sarcofuranocembrenolide B (22)

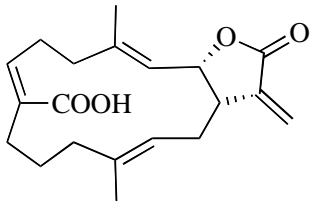

Lobohedleolide (25)

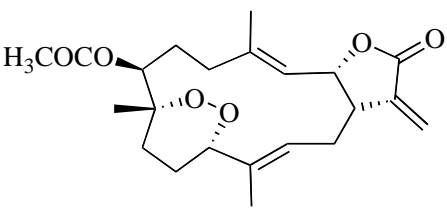

Denticulatolide (27)

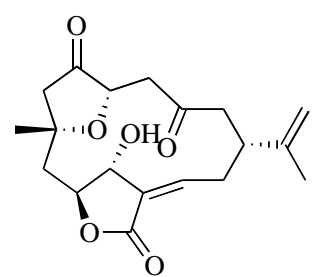

5-epi-sinuleptolide (23)

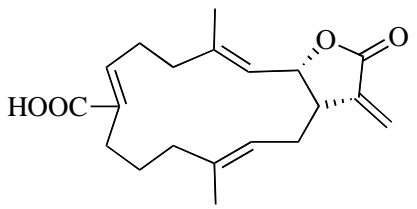

(7Z)-Lobohedleolide (26)
(25S)-3-Oxocholesta-1,4-dien-26-oic acid (17) and a new (25S)18-acetoxy-3-oxocholesta-1,4-dien-26-oic acid (18), together with two known cholic-acid-type compounds, 3-oxochol-1,4-dien-24oic acid (19) and 3-oxochol-4-en-24-oic acid (20), were isolated by Wang et al. from a soft coral Minabea sp. (cf. aldersladei) collected in North Sulawesi, Indonesia (Wang et al., 2009).

This is the first report of compound $\mathbf{1 7}$ as a natural product. Antimicrobial activity against Gram-positive ( $S$. aureus) and negative bacteria (E. coli), yeast (S. cerevisiae), and a
Filamentous fungus (M. hiemalis) and cytotoxicity against V79 and L1210 cells were examined, and compounds 17-20 showed no apparent activityat $100 \mu \mathrm{g} / \mathrm{disk}$ (antimicrobial), $10 \mu \mathrm{M}$ (V79 cells), and $50 \mu \mathrm{g} / \mathrm{mL}(\mathrm{L} 1210)$

Kapojos et al. reported two unusual cembranoids, sarcofuranocembrenolides A (21) and B (22), together with five known cembranoids named 5-epi-sinuleptolide (23),24, lobohedleolide(25), (7Z)-lobohedleolide (26) and denticulatolide (27) (Kapojos et al., 2010). Compounds 25-27 inhibited the 
colony formation of V79 cells at $\mathrm{ED}_{50}$ values of $4.6,3.7$, and 3.6 $\mathrm{mM}$, respectively, and reduced TNF $\alpha$ production from lipopolysaccharide (LPS)-stimulated mousemacrophage RAW 264.7 cells at $3.0-10.0 \mathrm{mM}$. Compounds $21-\mathbf{2 7}$ were isolated from a soft coral Sarcophyton sp., collected by scuba diving in Manado, North Sulawesi. Chen et al. described nine new diterpenoids named cladielloides A - B (28 -29), cladieunicellins $\mathrm{A}-\mathrm{F}(\mathbf{3 0}-\mathbf{3 5})$ and $(-)$-solenopodin $\mathrm{C}(\mathbf{3 6})$, which were isolated from an Indonesian octocoral identified as Cladiella sp (Chen et al., 2011).

Compound 29 exhibited moderate cytotoxicity toward CCRF-CEM tumor cells and this compound displayed significant inhibitory effects on superoxide anion generation and elastase release by human neutrophils. The cytotoxicity of metabolites 30-34 toward a limited panel of tumor cell lines, including DLD1, HL-60, CCRF-CEM (human T-cell acute lymphoblastic leukemia), and P388D1 cells was evaluated. The results showed that cladieunicellins B (31) and E (34) exhibited significant cytotoxicity against DLD-1 and HL-60 cells. Compound 32 displayed a significant inhibitory effect against superoxide anion generation by human neutrophils. Compound $\mathbf{3 6}$ displayed significant inhibitory effects on the generation of superoxide anion and the release of elastase by human neutrophils at a concentration of $10 \mathrm{mg} / \mathrm{mL}$. Similarly, from the same university, Tai et al. found two novel eunicellin-based diterpenoids, cladielloides C (37) and D (38) (Tai et al., 2011).

Compound $\mathbf{3 7}$ exhibited significant cytotoxicity toward CCRF-CEM tumor cells and metabolites $\mathbf{3 7}$ and $\mathbf{3 8}$ displayed moderate inhibitory effects on superoxide anion generation by human neutrophils. Anta et al. found two new xeniolides, xeniolide-F (39) and 9-hydroxyxeniolide-F (40), along with isoxeniolide-A (41) and 7,8-oxido-isoxeniolide-A (42), isolated from Xenia sp, collected in the Togian Islands near Sulawesi Island (Anta et al., 2002a). Compounds $39-\mathbf{4 2}$ showed an $\mathrm{IC}_{50}>1$ $\mathrm{g} / \mathrm{mL}$ against mouse (P-388) and human (A-549, HT-29, MEL-28) tumorcell lines. Fattorusso and colleagues also isolated four novel xenicane diterpenoids, xenimanadins A-D $(\mathbf{4 3}-\mathbf{4 6})$, characterized by the unusual 2, 6-dimethoxytetrahydropyran functionality, have been isolated from the Indonesian soft coral Xenia sp., together with three known xeniolides, named blumiolide $\mathrm{C}(\mathbf{4 7})$, xeniolide F (39), and deoxyoxidoisoxeniolide A (48) (Fattorusso et al., 2008). A new secosterol (49), along with pachyclavulariaenone B (50), was isolated from Pachyclavularia violacea by Anta et al. Compounds 49 and $\mathbf{5 0}$ showed an $\mathrm{IC}_{50}>1 \mu \mathrm{g} / \mathrm{mL}$ against mouse (P-388) and human (A-549, HT-29, MEL-28) tumor cell lines (Anta et al., 2002b).

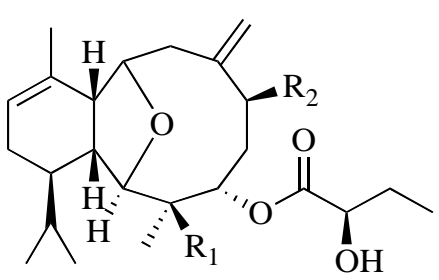

Cladielloide A (28) : R1= OAc, $\mathrm{R} 2=\mathrm{OH}$ Cladielloide B (29) : R1 = OH, R2 = OAc<smiles>CC1=CC[C@H](C(C)C)[C@]23O[C@H]1C[C@](C)(O)C(=O)C[C@@H](O)[C@H]2O3</smiles>

Cladieunicellin D (33)

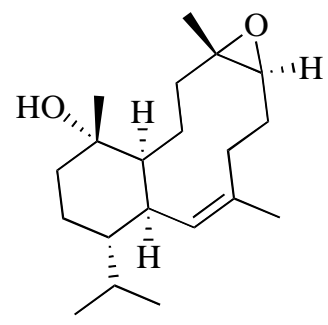

(-)-Solenopodin C (36)

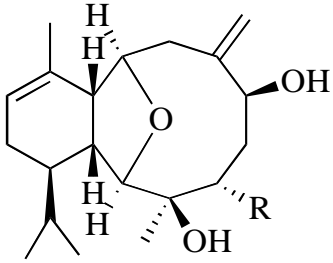

Cladieunicellin A (30) : R = H Cladieunicellin B (31) : $\mathrm{R}=\mathrm{OH}$

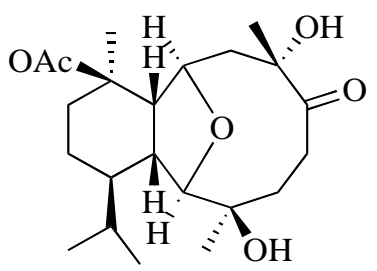

Cladieunicellin E (34)

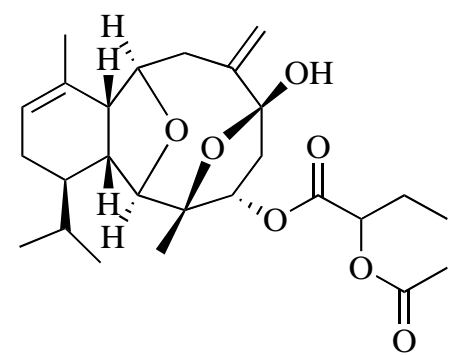

Cladielloide C (37)

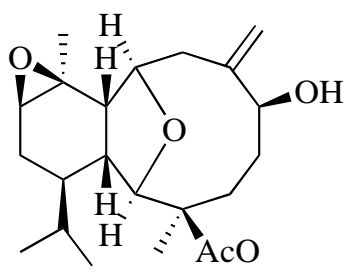

Cladieunicellin C (32)<smiles>C=C1CC[C@H]2[C@@H](O)CC[C@H](C(C)C)[C@H]2[C@H]2O[C@]2(O)CC[C@@H]1O</smiles>

Cladieunicellin F (35)<smiles>CC(=O)C[C@H]1O[C@H](C(C)(O)C=CC=O)[C@]2(C(C)C)C(C)=CC[C@H]1C2C</smiles>

Cladielloide D (38) 


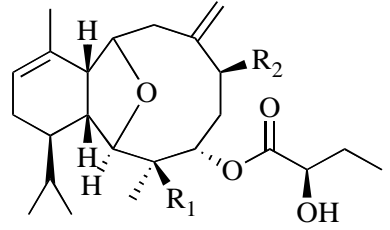

Cladielloide A (28) : R1= OAc, $\mathrm{R} 2=\mathrm{OH}$ Cladielloide B (29) : R1 = OH, R2 = OAc

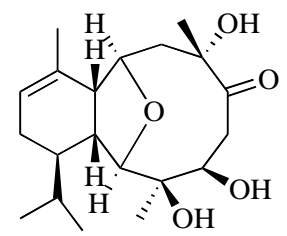

Cladieunicellin D (33)

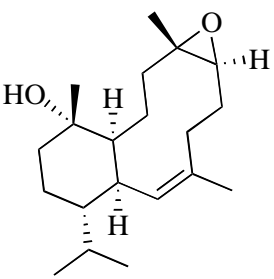

(-)-Solenopodin C (36)

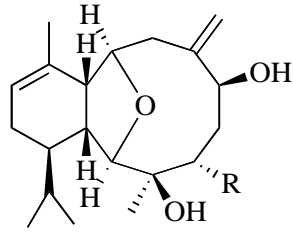

Cladieunicellin A (30) : $\mathrm{R}=\mathrm{H}$ Cladieunicellin B (31) : $\mathrm{R}=\mathrm{OH}$

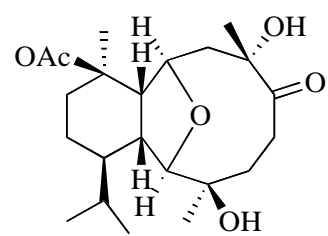

Cladieunicellin E (34)

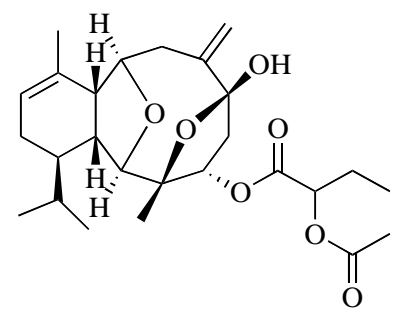

Cladielloide C (37)

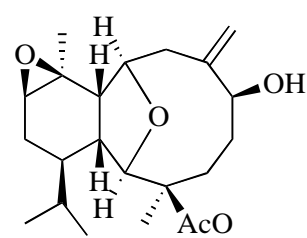

Cladieunicellin C (32)<smiles>C=C1CC[C@@H]2[C@@H]([C@@H](O)CC[C@@H]2C(C)C)C2(O)O[C@@H]1CC[C@@H]2O</smiles>

Cladieunicellin F (35)<smiles>CC(=O)C[C@H]1OC[C@H](C(C)O)[C@H](C(C)C)[C@H]1C=CC=O</smiles>

Cladielloide D (38)

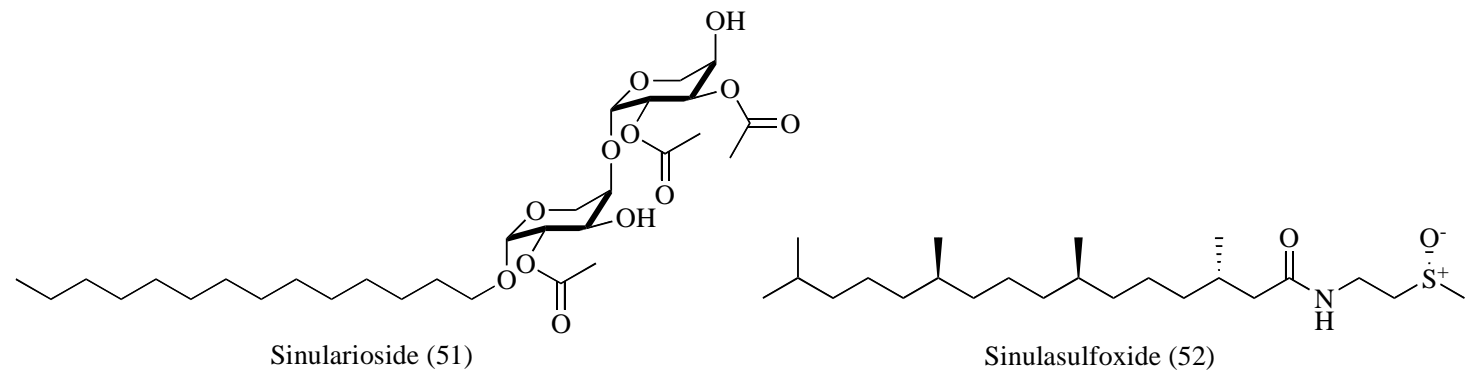

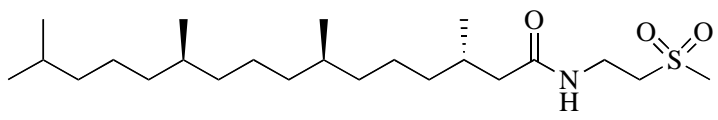

Sinulasulfone (53)
HOOC

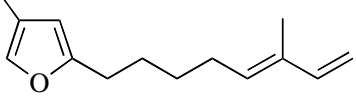

Furanosesquiterpenoid (54)
Putra and colleagues isolated three new compounds named sinularioside (51), sinulasulfoxide (52), sinulasulfone(53), along with two known compounds named furanosesquiterpenoid (54) and cerebroside (55) from Indonesian soft coral Sinularia sp collected in Siladen Island, Bunaken Marine National Park (North Sulawesi) (Putra et al., 2012a; Putra et al., 2012b). Compound 51 proved to consistently inhibit the expression of iNOS protein, a potentially anti-inflammatory activity. Putra et al. also isolated three new sterols $(56$ - 58) and five known sterols (59-63) (Putra et al., 2012c) characterized by either 24-methylcholestane or gorgostane skeletons. The isolated steroids have been evaluated for their interaction with the farnesoid X-activated receptor (FXR) and some of them, including the new compound $\mathbf{5 8}$ and gorgosterol (62), showed a consistent antagonistic activity, potentially useful for the treatment of cholestasis. The FXR antagonistic activity of gorgosterol was also supported by gene expression experiments. Compound $\mathbf{6 2}$ represents the first evaluation of soft coral steroids for interaction with nuclear receptors and qualify gorgosterol as a new chemotype of FXR antagonist. Similarly, from the same university, Fattorusso et al. isolated two known C-4 norcembranoids,, named leptocladolide B (64) and scabrolide D (65), and three new ones, named chloroscabrolides A (66) and B (67) and prescabrolide (68). All the norcembranoids were evaluated for anti-inflammatory activity, scabrolide D (65) at a concentration of $10 \mu \mathrm{M}$, a $15 \%$ inhibition of $\mathrm{NO}_{2}{ }^{-}$production was observed (Fattorusso et al., 2011). 

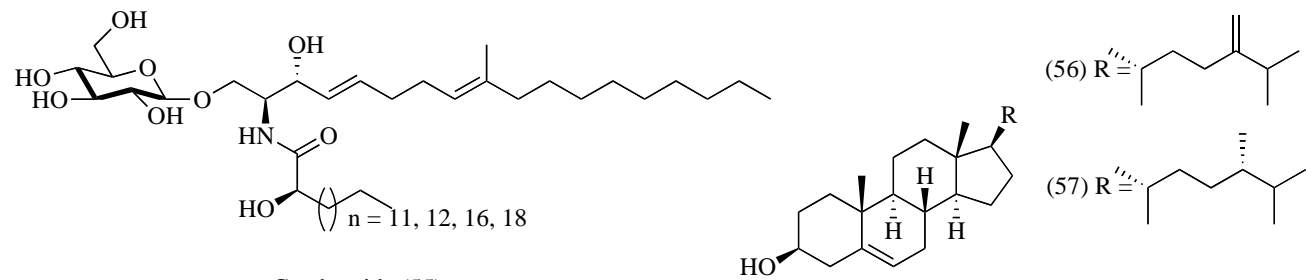

Cerebroside (55)
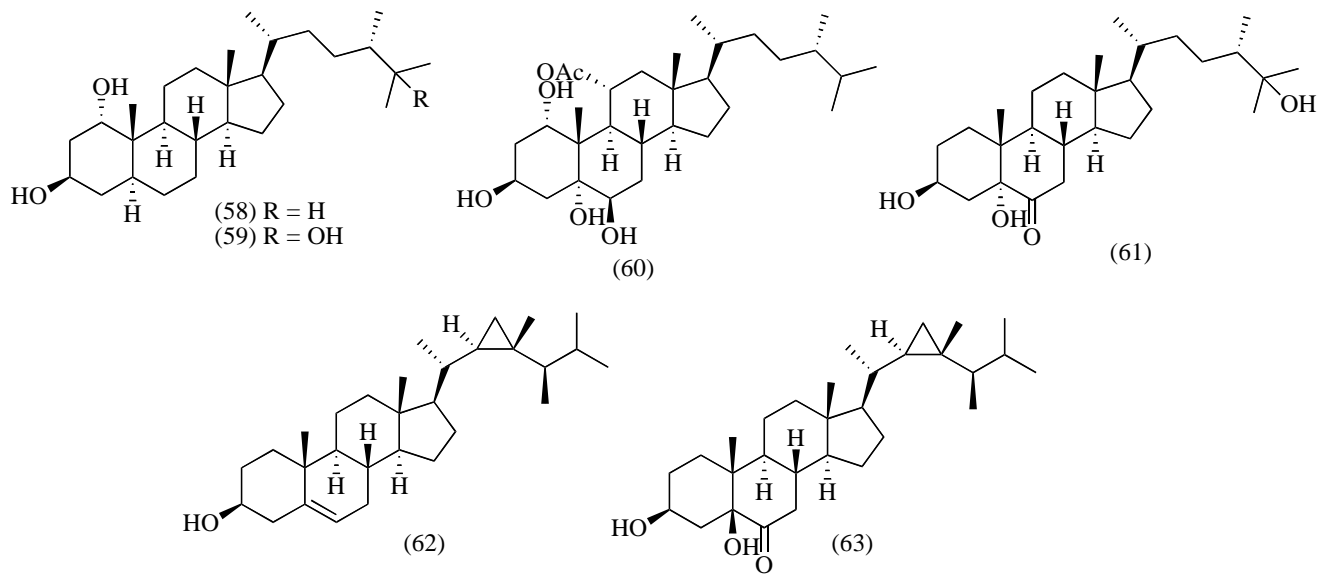

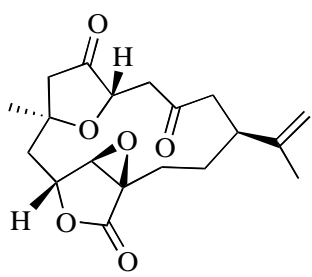

scabrolide D (64)

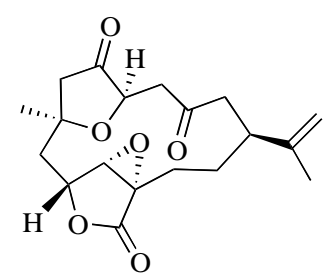

leptocladolide B (65)

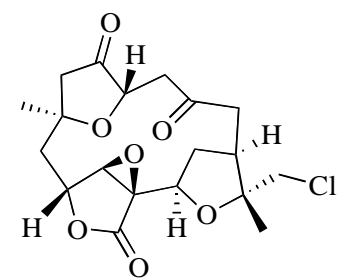

chloroscabrolide A (66)

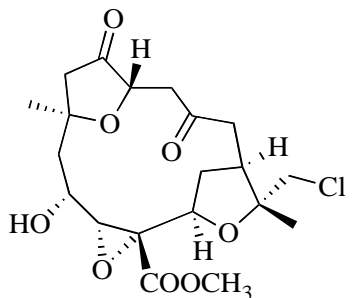

chloroscabrolide B (67)

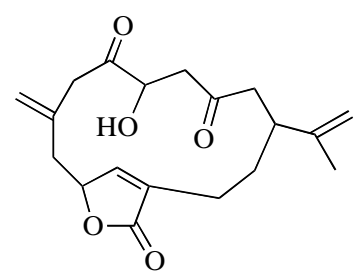

prescabrolide (68)

\section{Gorgonians}

Rodriguez et al. reported the Indonesian gorgonian Briareum sp. has afforded two new briarane stecholide diterpenes, named 2,9-diacetyl-2-debutyrylstecholide $\mathrm{H}$ (69) and 13-dehydroxystecholide $\mathbf{J}$ (70), the semisynthetic $2 \beta$-acetoxy-2(debutyryloxy) stecholide E acetate (71), which was isolated as a natural product for the first time, along with the known compounds stecholides I - M (72-76), stecholide A acetate (77), and stecholide $\mathrm{C}$ acetate (78) (Rodríguez et al., 1998).

Compound 71 was reported to exhibit significant activity against the growth of $\mathrm{P}-388$ cells $\left(\mathrm{EC}_{50} 1.59 \mu \mathrm{g} / \mathrm{mL}\right)$ and this is the first time that mild cytotoxic activity is found for the known compound 75. The gorgonian Briareum sp., was collected along the coast of the Togian Islands. Four new diterpenoids with the briarane skeleton, -epoxy-4- deacetoxyjunceellolide D (82) $\beta$, $20 \beta$-epoxy-4- deacetyljunceellolide D (81), and (-)-11 $\beta, 20 \beta$ epoxyjunceellolide D (80), (-)-11 $\alpha, 20 \alpha(-)-4$-deacetyljunceellolide D (79), (+)- 11, (+)-junceellolide A (83) [the antipodal derivative of the known (-)-junceellolide A], along with three known briaranes, (-)-junceellolide D (84), (-)-junceellin (85), and (-)praelolide (86), were isolated from the Indonesian gorgonian Junceella fragilis by García et al. (1999). Specimens of Junceella fragilis were collected at Halmahera Island. Gonzalez et al. isolated thirteen new Polyoxygenated steroids (87-99) along with the known compounds (100-103) from the Indonesian gorgonian Isis hippuris (González et al., 2011). 


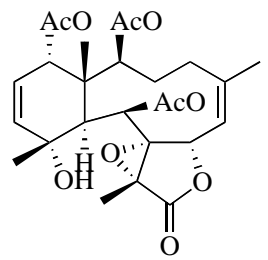

2,9-Diacetyl-2-debutyrylstecholide H (69)

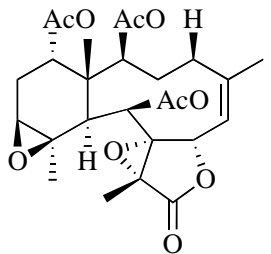

2b -Acetoxy-2-(debutyryloxy)stecholide E acetate (71)

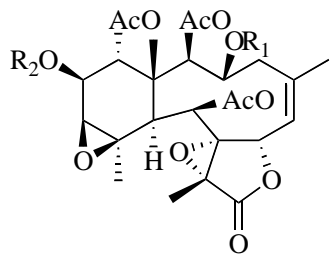

Stecholide $\mathrm{K}$ (74), $\mathrm{R}_{1}=\mathrm{R}_{2}=\mathrm{Ac}$ Stecholide $\mathrm{L}$ (75), $\mathrm{R}_{1}=\mathrm{Ac}, \mathrm{R}_{2}=\mathrm{H}$ Stecholide $M(76), \mathrm{R}_{1}=\mathrm{C}_{\mathrm{O}} \mathrm{C}_{3} \mathrm{H} 7, \mathrm{R}_{2}=\mathrm{H}$

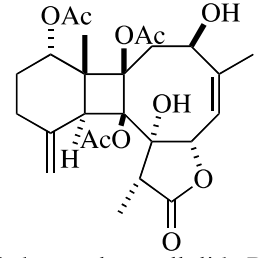

(-)-4-deacetylunceellolide D (79)

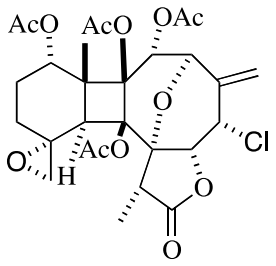

(-)-4-praeolide (86)

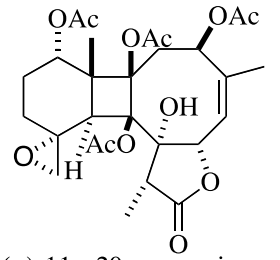

(+)-11 $\alpha, 20 \alpha$-epoxyjunceellolide D $(80)$

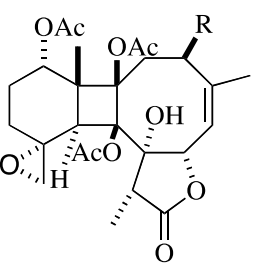

$\mathrm{O}$

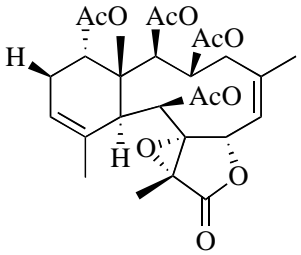

13-Dehydroxystecholide J (70)

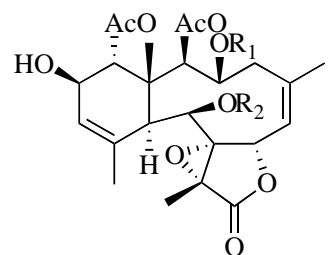

Stecholide I (72), $\mathrm{R}_{1}=\mathrm{COC}_{3} \mathrm{H}_{7}, \mathrm{R}_{2}=\mathrm{Ac}$ Stecholide $\mathrm{J}$ (73), $\mathrm{R}_{1}=\mathrm{R}_{2}=\mathrm{Ac}$

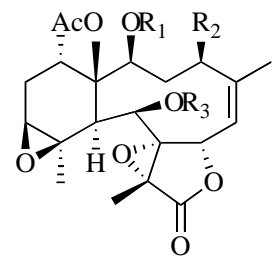

Stecholide A acetate (77), $\mathrm{R}_{1}=\mathrm{COC}_{3} \mathrm{H}_{7}, \mathrm{R}_{2}=\mathrm{OAc}, \mathrm{R}_{3}=\mathrm{Ac}$ Stecholide $\mathrm{C}$ acetate (78), $\mathrm{R}_{1}=\mathrm{R} 3=\mathrm{Ac}, \mathrm{R}_{2}=\mathrm{OAc}$

$$
\begin{aligned}
& \mathrm{R}=\mathrm{OH},(-)-11 \alpha, 20 \alpha \text {-epoxy-4-deacetyljunceellolide D (81) } \\
& \mathrm{R}=\mathrm{H},(-)-11 \alpha, 20 \alpha \text {-epoxy-4-deacetoxyjunceellolide D (82) }
\end{aligned}
$$

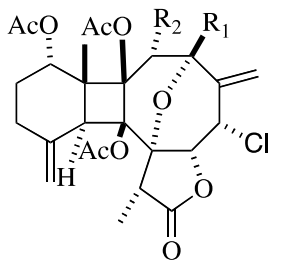

$\mathrm{R}_{1}=\mathrm{OH}, \mathrm{R}_{2}=\mathrm{H},(+)$-junceellolide A (83) $\mathrm{R}_{1}=\mathrm{H}, \mathrm{R}_{2}=\mathrm{OAc},(-)$-junceellin A (85)

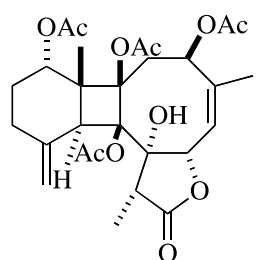

(-)-Junceellolide D (84)

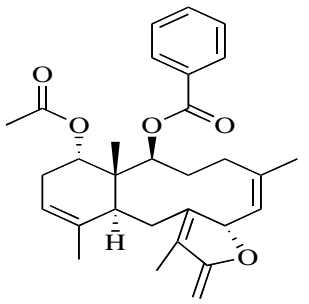

Malayenolides A (104)

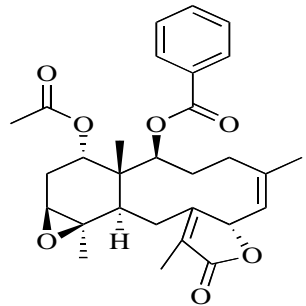

Malayenolides B (105)

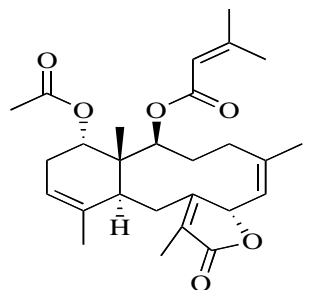

Malayenolides C (106)

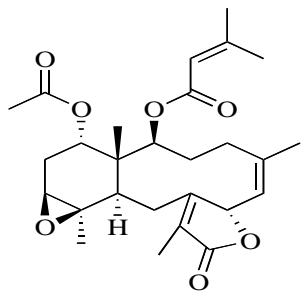

Malayenolides D (107) 


\section{Sea pen}

Four new briarane diterpenes, named Malayenolides A-D (104-107), were isolated from the sea pen Veretillum malayense collected in Indonesia. Malayenolides A-D (1-4) showed toxicity to brineshrimp, LC50 $100 \mu \mathrm{g} / \mathrm{mL},<2 \mu \mathrm{g} / \mathrm{mL}, 20 \mu \mathrm{g} / \mathrm{mL}$, respectively (Fu et al., 1999).

\section{CONCLUSION}

Marine invertebrates, which are plentiful in the IndoPacific regions including Indonesia, are rich in secondary metabolites and are becoming targets for the continuing search for bioactive compounds. The new metabolites from Indonesian marine organisms were mainly isolated from sponges. Octocorals are also being studied with promising results for secondary metabolites. Soft corals are the dominant reef dwelling octocorals in Indonesia. Since 1997-2014, more than 20 publications have reported on the bioactive compounds from Indonesian soft corals such as Cladiella sp., Lobophytum sp. and Sinularia sp. (Putra and Murniasih, 2016).

The discovery of novel compounds from Indonesian sea pens and gorgonians is much rarer, as indicated by the fact that until now only three publications have described novel compounds from them. Terpenes and sterols represented the two main chemical classes of compounds discovered from Indonesian marine organisms.

\section{ACKNOWLEDGMENT}

Financial support and sponsorship: This work was supported by a research grant from the Coral Reef Rehabilitation and Management Program-Coral Triangle Initiative (COREMAP CTILIPI 2016 No. 10876401/ ADB LOAN No. 3094 - INO), Indonesian Institute of Sciences awarded to Tutik Murniasih and Masteria Yunovilsa Putra.

Conflict of Interests: There are no conflicts of interest.

\section{REFERENCES}

Almeida MTR, Moritz MIG, Capel KCC, Pérez CD, Schenkel EP. Chemical and biological aspects of octocorals from the Brazilian coast. Rev Bras Farmacogn, 2014; 24: 446-467

Anta C, Gonzáles N, Santafé G, Rodríguez J, Jiménez C. New Xenia diterpenoids from the Indonesian soft coral Xenia sp. J Nat Prod, 2002a; 65: 766-768.

Anta C, Gonzáles N, Santafé G, Rodríguez J, Jiménez C. A new secosterol from the Indonesian octocoral Pachyclavularia violacea. J Nat Prod, 2002b; 65: 1357-1359.

Chen YH, Tai CY, Kuo YH, Kao CY, Li JJ, Hwang TL, Fang LS, Wang WH, Sheu JH, Sung PJ. Cladieunicellins A-E, New Eunicellins from an Indonesian Soft Coral Cladiella sp. Chem Pharm Bull, 2011; 59: 353-358

Daly M, Brugler MR, Cartwright P, Collins AG, Dawson MN, Fautin DG, France SC, McFadden CS, Opresko DM, Rodríguez E, Romano SL, Stake JL. The phylum Cnidaria: A review of phylogenetic patterns and diversity 300 years after Linnaeus. Zootaxa, 2007; 1668: 127 182
Ebada SS, ProkschP. 2012 The chemistry of marine sponges. In: Fattorusso E, Gerwick WH, Taglialatela-Scafati O, ed. Handbook of Marine natural products. Springer Netherlands; 191-293.

Fattorusso E, Luciano P, Putra MY, Taglialatela-Scafati O, Ianaro A, Panza E, Bavestrello G, Cerrano C. Chloroscabrolides, chlorinated norcembranoids from the Indonesian soft coral Sinularia sp. Tetrahedron, 2011; 67: 7983-8.

Fattorusso E, Romano A, Taglialatela-Scafati O, Irace $O$, Maffettone C, Bavesterllo G, Cerrano C. Oxygenated cembranoids of the decaryiol type from the Indonesian soft coral Lobophytum sp. Tetrahedron, 2009; 65: 2898-2904.

Fattorusso E, Romano A, Taglialatela-Scafati O, Achmad MJ, Bavesterllo G, Cerrano C. Xenimanadins AeD, a family of xenicane diterpenoidsfrom the Indonesian soft coral Xenia sp. Tetrahedron, 2008; 64: 3141-3146.

Fu X, Schmitz FJ, Williams GC. Malayenolides A-D, novel diterpenes from the Indonesian sea pen Veretillum malayense. J Nat Prod, 1999; 62: 584-586.

García M, Rodríguez J, Jiménez C. Absolute structures of new briarane diterpenoids from Junceella fragilis. J Nat Prod, 1999; 62: 257 260

González N, Barral MA, Rodríguez J, Jiménez C. New cytotoxic steroids from the gogonian Isis hippuris. Structure-activity studies. Tetrahedron, 2001; 57: 3487-3497

Handayani D, Edrada RA, Proksch P, Wray V, Witte L, Ofwegen LV, Kunzmann A. New oxygenated sesquiterpenoid from the Indonesian Soft coral Nepthea chabroli. J Nat Prod, 1997; 60: 716-718

Januar HI, Chasanah E, Motti CA, Tapiolas DM, Liptrot CH, Wright AD. Cytotoxic cembranes from Indonesian specimens of the soft coral Nephthea sp. Mar Drugs, 2010; 8: 2142-2152.

Kapojos MM, Lee JS, Oda T, Nakazawa T, Takahashi O, Ukai $\mathrm{K}$, Mangindaa REP, Rotinsulu $\mathrm{H}$, Wewengkang DS, Tsukamoto S, Kobayashi H, Namikoshi M. Two unprecedented cembrane-type terpenes from an Indonesian soft coral Sarcophyton sp. Tetrahedron, 2010; 66: 641645.

McFadden CS, Sánchez JA, France SC. Molecular phylogenetic insights into the evolution of Octocorallia: a review. Integr Comp Biol, 2010; 50: 389-410.

Molinski TF, Dalisay DS, Lievens SL, Saludes JP. Drug development from marine natural products. Nat Rev Drug Discov, 2009; 8: 69-85.

Morris LA, Christie M, Jaspars M, Ofwegen LPV. A bioactive secosterol with an Unusual A- and B-ring Oxygenation pattern isolated from an Indonesian soft coral Lobophytum sp. J Nat Prod, 1998; 61: 538541.

Putra MY, Ianaro A, Panza E, Bavestrello G, Cerrano C, Fattorusso E, Taglialatela-Scafati O. Sinularioside, a triacetylated glycolipid from the Indonesian soft coral Sinularia sp., is an inhibitor of NO release. Bioorg Med Chem Lett, 2012a; 22: 2723-5.

Putra MY, Ianaro A, Panza E, Bavestrello G, Cerrano C, Fattorusso E, Taglialatela-Scafati O.Sinulasulfoxide and sinulasulfone, new sulfur-containing alkaloidsfrom the indonesian soft coral Sinularia sp. Tetrahedron Lett, 2012b; 53:3937-9.

Putra MY, Bavestrello G, Cerrano C, Renga B, D'Amore C, Fiorucci S, Fattorusso E, Taglialatela-Scafati O. Polyhydroxylated sterols from the Indonesian soft coral Sinularia sp. and their effect on farnesoid X-activated receptor. Steroids, 2012c; 77: 433-440

Putra MY, Murniasih T. Distribution and diversity of marine natural products from Indonesian marine organisms. J Coast Life Med, 2016; 4(2): 104-107

Rocha J, Calado R, Leal M.2015.Marine bioactive compounds from cnidarians. In: Se-Kwon Kim, ed. Springer Handbook of Marine Biotechnology. Springer Berlin Heidelberg. pp.823-849

Rocha J, Peixe L, Gomes NCM, Calado R. Cnidarians as a source of new marine bioactive compounds - an overview of the last decade and future steps for bioprospecting. Mar Drugs, 2011; 9: 18601886. 
Rodríguez J, Nieto RM, Jimenez C. New briarane stecholide diterpenes from the Indonesian Gorgonian Briareum sp. J Nat Prod, 1998; 61: 313-317.

Tai CY, Chen YH, Hwang TL, Fang LS, Wang WH, Liu MC, $\mathrm{Su}$ JH, Wu YC, Sung PJ. Cladielloides C and D: Novel eunicellin-based diterpenoids from an Indonesian octocoral Cladiella sp. Bull Chem Soc Jpn, 2011; 84: 531-536.

Wang W, Lee JS, Nakazawa T, Ukai K, Mangindaan REP, Wewengkang DS, Rotinsulu H, Kobayashi H, Tsukamoto S, Namikoshi M. (25S)-Cholesten-26-oic acid derivatives from an Indonesian soft coral Minabea sp. Steroids, 2009; 74: 758-760.

\section{How to cite this article:}

Putra MY, Wibowo JT, Murniasih T. A review of chemistry and biological activities of the Indonesian Octocorallia. J App Pharm Sci, 2017; 7 (05): 219-227. 